\title{
STATISTICAL CONVERGENCE OF MARTINGALE DIFFERENCE SEQUENCE VIA DEFERRED WEIGHTED MEAN AND KOROVKIN-TYPE THEOREMS
}

\author{
BIDU BHUSAN JENA AND SUSANTA KUMAR PAIKRAY \\ Received 07 September, 2020
}

\begin{abstract}
In the present paper, we introduce and study the concepts of statistical convergence and statistical summability for martingale difference sequences of random variables via deferred weighted summability mean. We then establish an inclusion theorem concerning the relation between these two beautiful concepts. Also, based upon our proposed notions, we state and prove new Korovkin-type approximation theorems with algebraic test functions for a martingale difference sequence over a Banach space and demonstrate that our theorems effectively extend and improves most (if not all) of the previously existing results (in statistical and classical versions). Finally, we present an illustrative example by using the generalized Bernstein polynomial of a martingale difference sequence in order to demonstrate that our established theorems are stronger than its traditional and statistical versions.
\end{abstract}

2010 Mathematics Subject Classification: 40A05; 40G15; 41A36

Keywords: stochastic sequences, martingale difference sequences, statistical convergence of martingale difference sequences, deferred weighted mean, Banach space, Korovkin-type theorem, Bernstein polynomial and positive linear operators

\section{INTRODUCTION AND MOTIVATION}

Let $(\Omega, \mathcal{F}, \mathbb{P})$ be a probability measurable space and suppose that $\left(Y_{n}\right)$ be a difference random variable such that $Y_{n}=X_{n}-X_{n-1}$ defined over this space, where $\left(X_{n}\right)$ and $X_{n-1}$ are also random variables belongs to this space. Also, let $\mathcal{F}_{n} \subseteq \mathcal{F}(n \in \mathbb{N})$ be a monotonically increasing sequence of $\sigma$-fields of measurable sets. Now, considering the random variable $\left(Y_{n}\right)$ and the measurable functions $\left(\mathcal{F}_{n}\right)$, we adopt a stochastic sequence $\left(Y_{n}, \mathcal{F}_{n} ; n \in \mathbb{N}\right)$.

A given stochastic sequence $\left(Y_{n}, \mathcal{F}_{n} ; n \in \mathbb{N}\right)$ is said to be a martingale difference sequence if

(i) $\mathbb{E}\left|Y_{n}\right|<\infty$,

(ii) $\mathbb{E}\left(Y_{n+1} \mid \mathcal{F}_{n}\right)=0$ almost surely (a.s.) and

(iii) $Y_{n}$ is a measurable function of $\mathcal{F}_{1}, \mathcal{F}_{2}, \cdots, \mathcal{F}_{n}$,

where $\mathbb{E}$ is the mathematical expectation. 
Next, we discuss about the above properties of martingale difference sequence of random variables.

Suppose $\left(X_{n}\right)$ is a martingale sequence with respect to $\mathcal{F}_{n}$. Also, let

$$
Y_{n}=X_{n}-X_{n-1}, \quad n=2,3, \cdots
$$

Now,

$$
\mathbb{E}\left|Y_{n}\right| \leqq \mathbb{E}\left|X_{n}\right|+\mathbb{E}\left|X_{n-1}\right|<\infty
$$

Next,

$$
\begin{aligned}
\mathbb{E}\left(Y_{n+1} \mid \mathcal{F}_{n}\right) & =\mathbb{E}\left(X_{n+1}-X_{n} \mid \mathcal{F}_{n}\right) \\
& =\mathbb{E}\left(X_{n+1} \mid \mathcal{F}_{n}\right)-X_{n} \quad\left(\because X_{n} \text { is a constant on } \mathcal{F}_{n}\right) \\
& =0 \quad\left(\because \mathbb{E}\left(X_{n+1} \mid \mathcal{F}_{n}\right)=X_{n}\right) .
\end{aligned}
$$

Since, $\left(X_{n}\right)$ and $\left(X_{n-1}\right)$ are measurable, therefore $\left(Y_{n}\right)$ is measurable.

We now recall the definition for convergence of martingale difference sequences of random variables.

Definition 1. A martingale difference sequence $\left(Y_{n}, \mathcal{F}_{n} ; n \in \mathbb{N}\right)$ with $\mathbb{E}\left|Y_{n}\right|$ is bounded and $\operatorname{Prob}\left(Y_{n}\right)=1$ (that is, with probability 1 ) is said to be convergent to a martingale $\left(Y_{0}, \mathcal{F}_{0}\right)$, if

$$
\lim _{n \rightarrow \infty}\left(Y_{n}, \mathcal{F}_{n}\right) \longrightarrow\left(Y_{0}, \mathcal{F}_{0}\right) \quad\left(\mathbb{E}\left|Y_{0}\right|<\infty\right) .
$$

The notion of statistical convergence has been one of the beautiful aspects of the sequence space theory and such an interesting notion was introduced by Fast [5]. Subsequently, the notion of probability convergence for sequences of random variables was introduced and such a notion is more general than the statistical convergence as well as of the usual convergence. Using both the concepts with different settings, various researchers developed many interesting results in several fields of pure and applied mathematics such as summability theory, Fourier series, approximation theory, probability theory, measure theory and so on, see $[2,3,7-9,12,15,18,19]$ and [23].

Let $\mathfrak{X} \subseteq \mathbb{N}$, and also let $\mathfrak{X}_{n}=\{j: j \leqq n$ and $\quad j \in \mathfrak{X}\}$. Then the natural density $d(\mathfrak{X})$ of $\mathfrak{X}$ is defined by

$$
d(\mathfrak{X})=\lim _{n \rightarrow \infty} \frac{\left|\mathfrak{X}_{n}\right|}{n}=\chi,
$$

where $\chi$ is real and a finite number, and $\left|\mathfrak{X}_{n}\right|$ is the cardinality of $\mathfrak{X}_{n}$.

We now recall the definition of statistical convergence for real sequence.

Definition 2 (see [5]). A given sequence $\left(u_{n}\right)$ is statistically convergent to $\kappa$ if, for each $\varepsilon>0$,

$$
\mathfrak{X}_{\varepsilon}=\left\{j: j \in \mathbb{N} \quad \text { and } \quad\left|u_{j}-\kappa\right| \geqq \varepsilon\right\}
$$


has zero natural density. Thus, for each $\varepsilon>0$, we have

$$
d\left(\mathfrak{X}_{\varepsilon}\right)=\lim _{n \rightarrow \infty} \frac{\left|\mathfrak{X}_{\varepsilon}\right|}{n}=0 .
$$

Here, we write

$$
\text { stat } \lim _{n \rightarrow \infty} u_{n}=\kappa .
$$

We now introduce the definition of statistical convergence of martingale difference sequence for random variables.

Definition 3. A bounded martingale difference sequence $\left(Y_{n}, \mathcal{F}_{n} ; n \in \mathbb{N}\right)$ having probability 1 is said to be statistically convergent to a martingale $\left(Y_{0}, \mathcal{F}_{0}\right)$ with $\mathbb{E}\left|Y_{0}\right|<\infty$ if, for all $\varepsilon>0$,

$$
\mathfrak{R}_{\varepsilon}=\left\{j: j \leqq n \quad \text { and } \quad\left|\left(Y_{j}, \mathcal{F}_{j}\right)-\left(Y_{0}, \mathcal{F}_{0}\right)\right| \geqq \varepsilon\right\}
$$

has zero natural density. That is, for every $\varepsilon>0$, we have

$$
d\left(\Re_{\varepsilon}\right)=\lim _{n \rightarrow \infty} \frac{\left|\Re_{\varepsilon}\right|}{n}=0 .
$$

Here, we write

$$
\operatorname{stat}_{\mathrm{MD}} \lim _{n \rightarrow \infty}\left(Y_{n}, \mathcal{F}_{n}\right)=\left(Y_{0}, \mathcal{F}_{0}\right) .
$$

Now we present an example illustrating that every martingale difference convergent sequence is statistically convergent, but not conversely.

Example 1. Let $\left(\mathcal{F}_{n} ; n \in \mathbb{N}\right)$ be a monotonically increasing sequence of 0-mean independent random variables over $\sigma$-fields and suppose $\left(X_{n}\right)$ is a sequence of $n$th partial sum of $\left(\mathcal{F}_{n} ; n \in \mathbb{N}\right)$ such that $X_{n}-X_{n-1}=Y_{n}$. Consider the sequence of random variables $\left(X_{n}\right)$ as

$$
X_{n}= \begin{cases}1 & \left(n=m^{2} ; m \in \mathbb{N}\right) \\ 0 & \text { (otherwise) }\end{cases}
$$

It is easy to see that, the martingale difference sequence $\left(Y_{n}, \mathcal{F}_{n} ; n \in \mathbb{N}\right)$ is statistically convergent to zero but not simply martingale difference convergent.

Based on our proposed definition, we establish a theorem concerning a relation between ordinary and statistical versions of convergence of martingale difference sequences.

Theorem 1. If a martingale difference sequence $\left(Y_{n}, \mathcal{F}_{n} ; n \in \mathbb{N}\right)$ is convergent to a martingale $\left(Y_{0}, \mathcal{F}_{0}\right)$ with $\mathbb{E}\left|Y_{0}\right|<\infty$, then it is statistically convergent to the same martingale. 
Proof. Let the martingale difference sequence $\left(Y_{n}, \mathcal{F}_{n} ; n \in \mathbb{N}\right)$ be bounded and converges with probability 1 , then there exists a martingale $\left(Y_{0}, \mathcal{F}_{0}\right)$ with $\mathbb{E}\left|Y_{0}\right|<\infty$, that is

$$
\lim _{n \rightarrow \infty}\left(Y_{n}, \mathcal{F}_{n}\right) \longrightarrow\left(Y_{0}, \mathcal{F}_{0}\right) .
$$

As the given martingale sequence $\left(Y_{n}, \mathcal{F}_{n} ; n \in \mathbb{N}\right)$ is bounded with probability 1 , then for every $\varepsilon>0$, we have

$$
\frac{1}{n}\left\{j: j \leqq n \quad \text { and } \quad\left|\left(Y_{j}, \mathcal{F}_{j}\right)-\left(Y_{0}, \mathcal{F}_{0}\right)\right| \geqq \varepsilon\right\} \subseteq \lim _{n \rightarrow \infty}\left|\left(Y_{n}, \mathcal{F}_{n}\right)-\left(Y_{0}, \mathcal{F}_{0}\right)\right|<\varepsilon .
$$

Consequently, by Definition 3, we obtain

$$
\frac{1}{n}\left\{j: j \leqq n \quad \text { and } \quad\left|\left(Y_{j}, \mathcal{F}_{j}\right)-\left(Y_{0}, \mathcal{F}_{0}\right)\right| \geqq \varepsilon\right\}=0 .
$$

Motivated essentially by the above mentioned investigations, we introduce and study the concepts of statistical convergence and statistical summability for martingale difference sequences of random variables via deferred weighted summability mean. We then establish an inclusion theorem concerning the relation between these two beautiful concepts. Also, based upon our proposed notions, we state and prove new Korovkin-type approximation theorems with algebraic test functions for a martingale difference sequence over a Banach space and demonstrate that our theorems effectively extend and improves most (if not all) of the previously existing results (in statistical and classical versions). Finally, we present an illustrative example by using the generalized Bernstein polynomial of a martingale difference sequence in order to demonstrate that our established theorems are stronger than its traditional and statistical versions.

\section{Deferred Weighted Martingale Difference Sequence}

Let $\left(a_{n}\right)$ and $\left(b_{n}\right)$ be sequences of non-negative integers such that $a_{n}<b_{n}$ and $\lim _{n \rightarrow \infty} b_{n}=+\infty$, and let $\left(p_{i}\right)$ be a sequence of non-negative numbers such that

$$
P_{n}=\sum_{i=a_{n}+1}^{b_{n}} p_{i}
$$

Then the deferred weighted mean for the martingale difference sequence $\left(Y_{n}, \mathcal{F}_{n} ; n \in \mathbb{N}\right)$ of random variables is defined by

$$
\mathfrak{W}\left(Y_{n}, \mathcal{F}_{n}\right)=\frac{1}{P_{n}} \sum_{i=a_{n}+1}^{b_{n}} p_{i}\left(Y_{i}, \mathcal{F}_{i}\right)
$$

It will be interesting to see that, for $p_{i}=1, \mathfrak{W}\left(Y_{n}, \mathcal{F}_{n}\right)$ reduces to deferred Cesàro mean $\left\{\mathcal{D}\left(X_{n}, \mathcal{F}_{n}\right): X_{n}=\sum_{i=1}^{n} Y_{i}\right\}$ which has been recently introduced by Srivastava 
et al. [17]. Moreover, recalling another result of Srivastava et al. [20] via deferred Nörlund mean $D_{a}^{b}(N, p, q)$ for real sequence given by

$$
t_{n}=\frac{1}{\mathcal{R}_{a}} \sum_{m=a_{n}+1}^{b_{n}} p_{b_{n}-m} q_{m} x_{m},
$$

one can also extend the same for the martingale difference sequence.

We now present the definitions of deferred weighted statistical convergence and statistically deferred weighted summability of martingale difference sequences of random variables.

Definition 4. Let $\left(a_{n}\right)$ and $\left(b_{n}\right)$ be sequences of non-negative integers, and let $\left(p_{n}\right)$ be a sequence of non-negative numbers. A bounded martingale difference sequence $\left(Y_{n}, \mathcal{F}_{n} ; n \in \mathbb{N}\right)$ of random variables having probability 1 is deferred weighted statistically convergent to a martingale $\left(Y_{0}, \mathcal{F}_{0}\right)$ with $\mathbb{E}\left|Y_{0}\right|<\infty$ if, for all $\varepsilon>0$,

$$
\mathfrak{Y}_{\varepsilon}=\left\{j: j \leqq P_{n} \quad \text { and } \quad p_{j}\left|\left(Y_{j}, \mathcal{F}_{j}\right)-\left(Y_{0}, \mathcal{F}_{0}\right)\right| \geqq \varepsilon\right\}
$$

has zero natural density. That is, for every $\varepsilon>0$, we have

$$
\lim _{n \rightarrow \infty} \frac{1}{P_{n}} \mid\left\{j: j \leqq P_{n} \quad \text { and } \quad p_{j}\left|\left(Y_{j}, \mathcal{F}_{j}\right)-\left(Y_{0}, \mathcal{F}_{0}\right)\right| \geqq \varepsilon\right\} \mid=0 .
$$

We write

$$
\mathrm{DWMD}_{\text {stat }} \lim _{n \rightarrow \infty}\left(Y_{n}, \mathcal{F}_{n}\right)=\left(Y_{0}, \mathcal{F}_{0}\right) .
$$

Definition 5. Let $\left(a_{n}\right)$ and $\left(b_{n}\right)$ be sequences of non-negative integers, and let $\left(p_{n}\right)$ be a sequence of non-negative numbers. A bounded martingale difference sequence $\left(Y_{n}, \mathcal{F}_{n} ; n \in \mathbb{N}\right)$ of random variables having probability 1 is statistically deferred weighted summable to a martingale $\left(Y_{0}, \mathcal{F}_{0}\right)$ with $\mathbb{E}\left|Y_{0}\right|<\infty$ if, for all $\varepsilon>0$,

$$
\mathfrak{Z}_{\varepsilon}=\left\{j: a_{n}<j \leqq b_{n} \quad \text { and } \quad\left|\mathfrak{W}\left(Y_{j}, \mathcal{F}_{j}\right)-\left(Y_{0}, \mathcal{F}_{0}\right)\right| \geqq \varepsilon\right\}
$$

has zero natural density. That is, for every $\varepsilon>0$, we have

$$
\lim _{n \rightarrow \infty} \frac{\mid\left\{j: a_{n}<j \leqq b_{n} \quad \text { and } \quad\left|\mathfrak{W}\left(Y_{j}, \mathcal{F}_{j}\right)-\left(Y_{0}, \mathcal{F}_{0}\right)\right| \geqq \varepsilon\right\} \mid}{b_{n}-a_{n}}=0 .
$$

We write

$$
\operatorname{stat}_{\text {DWMD }} \lim _{n \rightarrow \infty} \mathfrak{W}\left(Y_{j}, \mathcal{F}_{j}\right)=\left(Y_{0}, \mathcal{F}_{0}\right) .
$$

Now we establish an inclusion theorem concerning the above mentioned two new interesting definitions.

Theorem 2. If a given martingale difference sequence $\left(Y_{n}, \mathcal{F}_{n} ; n \in \mathbb{N}\right)$ of random variables is deferred weighted statistically convergent to a martingale $\left(Y_{0}, \mathcal{F}_{0}\right)$ with $\mathbb{E}\left|Y_{0}\right|<\infty$, then it is statistically deferred weighted summable to the same martingale, but not conversely. 
Proof. Suppose the given martingale sequence $\left(Y_{n}, \mathcal{F}_{n} ; n \in \mathbb{N}\right)$ of random variables is deferred weighted statistically convergent to a martingale $\left(Y_{0}, \mathcal{F}_{0}\right)$ with $\mathbb{E}\left|Y_{0}\right|<\infty$, then by Definition 4, we have

$$
\lim _{n \rightarrow \infty} \frac{1}{P_{n}} \mid\left\{j: j \leqq P_{n} \quad \text { and } \quad p_{j}\left|\left(Y_{j}, \mathcal{F}_{j}\right)-\left(Y_{0}, \mathcal{F}_{0}\right)\right| \geqq \varepsilon\right\} \mid=0 .
$$

Now assuming two sets as follows:

$$
\mathcal{W}_{\varepsilon}=\left\{j: j \leqq P_{n} \quad \text { and } \quad p_{j}\left|\left(Y_{j}, \mathcal{F}_{j}\right)-\left(Y_{0}, \mathcal{F}_{0}\right)\right| \geqq \varepsilon\right\}
$$

and

$$
\mathcal{W}_{\varepsilon}^{c}=\left\{j: j \leqq P_{n} \quad \text { and } \quad p_{j}\left|\left(Y_{j}, \mathcal{F}_{j}\right)-\left(Y_{0}, \mathcal{F}_{0}\right)\right|<\varepsilon\right\}
$$

we have

$$
\begin{aligned}
\left|\mathfrak{W}\left(Y_{n}, \mathcal{F}_{n}\right)-\left(Y_{0}, \mathcal{F}_{0}\right)\right|= & \left|\frac{1}{P_{n}} \sum_{i=a_{n}+1}^{b_{n}} p_{i}\left(Y_{i}, \mathcal{F}_{i}\right)-\left(Y_{0}, \mathcal{F}_{0}\right)\right| \\
\leqq & \frac{1}{P_{n}} \sum_{i=a_{n}+1}^{b_{n}} p_{i}\left[\left(Y_{i}, \mathcal{F}_{i}\right)-\left(Y_{0}, \mathcal{F}_{0}\right)\right] \mid \\
& +\left|\frac{1}{P_{n}} \sum_{i=a_{n}+1}^{b_{n}} p_{i}\left(Y_{0}, \mathcal{F}_{0}\right)-\left(Y_{0}, \mathcal{F}_{0}\right)\right| \\
\leqq & \frac{1}{P_{n}} \sum_{i=a_{n}+1}^{b_{n}}\left|p_{i}\left(Y_{i}, \mathcal{F}_{i}\right)-\left(Y_{0}, \mathcal{F}_{0}\right)\right| \\
& +\frac{1}{P_{n}} \sum_{i=\mathcal{W}_{\varepsilon}+1}^{b_{n}}\left|p_{i}\left(Y_{i}, \mathcal{F}_{i}\right)-\left(Y_{0}, \mathcal{F}_{0}\right)\right| \\
& +\left|\left(Y_{0}, \mathcal{F}_{0}\right)\right|\left|\frac{1}{P_{n}} \sum_{i=a_{n}+1}^{b_{n}} p_{i}-1\right| \\
\leqq & \frac{1}{P_{n}}\left|\mathcal{W}_{\mathcal{\varepsilon}}\right|+\frac{1}{P_{n}}\left|\mathcal{W}_{\varepsilon}^{c}\right|=0 .
\end{aligned}
$$

Clearly, we obtain

$$
\left|\mathfrak{W}\left(Y_{n}, \mathcal{F}_{n}\right)-\left(Y_{0}, \mathcal{F}_{0}\right)\right|<\varepsilon .
$$

Thus, the martingale difference sequence $\left(Y_{n}, \mathcal{F}_{n} ; n \in \mathbb{N}\right)$ of random variables is statistically deferred weighted summable to the martingale $\left(Y_{0}, \mathcal{F}_{0}\right)$ with $\mathbb{E}\left|Y_{0}\right|<\infty$.

Next, in support of the non-validity of the converse statement, we present here an example demonstrating that a statistically deferred weighted summable martingale difference sequence of random variables is not necessarily deferred weighted statistically convergent. 
Example 2. Suppose that $a_{n}=2 n, b_{n}=4 n$ and $p_{n}=n$, and let $\left(\mathcal{F}_{n} ; n \in \mathbb{N}\right)$ be a monotonically increasing sequence of 0 -mean independent random variables of $\sigma$ fields and suppose that $\left(X_{n}\right)$ is a sequence of $n$th partial sum of $\left(\mathcal{F}_{n} ; n \in \mathbb{N}\right)$ such that $X_{n}-X_{n-1}=Y_{n}$. Consider the sequence of random variables $\left(X_{n}\right)$ as

$$
X_{n}= \begin{cases}1 & (n=\text { even }) \\ -1 & (n=\text { odd }) .\end{cases}
$$

It is easy to see that, the martingale difference sequence $\left(Y_{n}, \mathcal{F}_{n} ; n \in \mathbb{N}\right)$ is neither convergent nor deferred weighted statistically convergent; however, it is deferred weighted summable to 0 . Therefore, it is statistically deferred weighted summable to 0 .

\section{A Korovkin-type Theorem for Martingale Difference SEquence}

Quite recently, a few researchers worked toward extending (or generalizing) the approximation of Korovkin-type theorems in different fields of mathematics such as sequence space, Banach space, Probability space, Measurable space, etc. This concept is extremely valuable in Real Analysis, Functional Analysis, Harmonic Analysis, and so on. Here, we like to refer the interested readers to the recent works $[4,13,18,20,21]$ and [26].

In fact, we establish here the statistical versions of new Korovin-type approximation theorems for martingale difference sequences of positive linear operators via deferred weighted summability mean.

Let $\mathcal{C}([0,1])$ be the space of all real valued continuous functions defined on $[0,1]$ under the norm $\|\cdot\|_{\infty}$. Also, let $\mathcal{C}[0,1]$ be a Banach space. Then for $f \in \mathcal{C}[0,1]$, the norm of $f$ denoted by $\|f\|$ is given by

$$
\|f\|_{\infty}=\sup _{x \in[0,1]}\{|f(x)|\} .
$$

We say that, an operator $\mathfrak{A}$ is a martingale difference sequence of positive linear operators provided that

$$
\mathfrak{A}(f ; x) \geqq 0 \quad \text { whenever } \quad f \geqq 0, \text { with } \mathfrak{A}(f ; x)<\infty \text { and } \operatorname{Prob}(\mathfrak{A}(f ; x))=1 .
$$

Theorem 3. Let

$$
\mathfrak{A}_{m}: \mathcal{C}[0,1] \rightarrow \mathcal{C}[0,1]
$$

be a martingale difference sequence of positive linear operators. Then, for all $f \in \mathcal{C}[0,1]$,

$$
\mathrm{DWMD}_{\text {stat }} \lim _{m \rightarrow \infty}\left\|\mathfrak{A}_{m}(f ; x)-f(x)\right\|_{\infty}=0
$$


if and only if

$$
\begin{aligned}
& \text { DWMD }_{\text {stat }} \lim _{m \rightarrow \infty}\left\|\mathfrak{A}_{m}(1 ; x)-1\right\|_{\infty}=0, \\
& \text { DWMD }_{\text {stat }} \lim _{m \rightarrow \infty}\left\|\mathfrak{A}_{m}(2 x ; x)-2 x\right\|_{\infty}=0
\end{aligned}
$$

and

$$
\text { DWMD }_{\text {stat }} \lim _{m \rightarrow \infty}\left\|\mathfrak{A}_{m}\left(3 x^{2} ; x\right)-3 x^{2}\right\|_{\infty}=0 .
$$

Proof. Since each of the following functions

$$
f_{0}(x)=1, \quad f_{1}(x)=2 x \quad \text { and } \quad f_{2}(x)=3 x^{2}
$$

belong to $C[0,1]$ and are continuous, the implication given by (3.1) implies (3.2) to (3.4) is obvious.

In order to complete the proof of the Theorem 3, we first assume that the conditions (3.2) to (3.4) hold true. If $f \in \mathcal{C}[0,1]$, then there exists a constant $\mathcal{N}>0$ such that

$$
|f(x)| \leqq \mathcal{N} \quad(\forall x \in[0,1]) .
$$

We thus find that

$$
|f(r)-f(x)| \leqq 2 \mathcal{N} \quad(r, x \in[0,1]) .
$$

Clearly, for given $\varepsilon>0$, there exists $\delta>0$ such that

$$
|f(r)-f(x)|<\varepsilon
$$

whenever

$$
|r-x|<\delta, \quad \text { for all } \quad r, x \in[0,1] .
$$

Let us choose

$$
\varphi_{1}=\varphi_{1}(r, x)=(2 r-2 x)^{2} .
$$

If $|r-x| \geqq \delta$, then we obtain

$$
|f(r)-f(x)|<\frac{2 \mathcal{N}}{\delta^{2}} \varphi_{1}(r, x) .
$$

From equation (3.6) and (3.7), we get

$$
|f(r)-f(x)|<\varepsilon+\frac{2 \mathcal{N}}{\delta^{2}} \varphi_{1}(r, x),
$$

which implies that

$$
-\varepsilon-\frac{2 \mathcal{N}}{\delta^{2}} \varphi_{1}(r, x) \leqq f(r)-f(x) \leqq \varepsilon+\frac{2 \mathcal{N}}{\delta^{2}} \varphi_{1}(r, x) .
$$

Now, since $\mathfrak{A}_{m}(1 ; x)$ is monotone and linear, by applying the operator $\mathfrak{A}_{m}(1 ; x)$ to this inequality, we have

$$
\mathfrak{A}_{m}(1 ; x)\left(-\varepsilon-\frac{2 \mathcal{N}}{\delta^{2}} \varphi_{1}(r, x)\right) \leqq \mathfrak{A}_{m}(1 ; x)(f(r)-f(x))
$$




$$
\leqq \mathfrak{A}_{m}(1 ; x)\left(\varepsilon+\frac{2 \mathcal{N}}{\delta^{2}} \varphi_{1}(r, x)\right) .
$$

We note that $x$ is fixed and so $f(x)$ is a constant number. Therefore, we have

$$
\begin{aligned}
-\varepsilon \mathfrak{A}_{m}(1 ; x)-\frac{2 \mathcal{N}}{\delta^{2}} \mathfrak{A}_{m}\left(\varphi_{1} ; x\right) & \leqq \mathfrak{A}_{m}(f ; x)-f(x) \mathfrak{A}_{m}(1 ; x) \\
& \leqq \varepsilon \mathfrak{A}_{m}(1 ; x)+\frac{2 \mathcal{N}^{2}}{\delta^{2}} \mathfrak{A}_{m}\left(\varphi_{1} ; x\right)
\end{aligned}
$$

Also, we know that

$$
\mathfrak{A}_{m}(f ; x)-f(x)=\left[\mathfrak{A}_{m}(f ; x)-f(x) \mathfrak{A}_{m}(1 ; x)\right]+f(x)\left[\mathfrak{A}_{m}(1 ; x)-1\right] .
$$

Using (3.9) and (3.10), we have

$$
\mathfrak{A}_{m}(f ; x)-f(x)<\varepsilon \mathfrak{A}_{m}(1 ; x)+\frac{2 \mathcal{A}}{\delta^{2}} \mathfrak{A}_{m}\left(\varphi_{1} ; x\right)+f(x)\left[\mathfrak{A}_{m}(1 ; x)-1\right] .
$$

We now estimate $\mathfrak{A}_{m}\left(\varphi_{1} ; x\right)$ as follows:

$$
\begin{aligned}
\mathfrak{A}_{m}\left(\varphi_{1} ; x\right)= & \mathfrak{A}_{m}\left((2 r-2 x)^{2} ; x\right)=\mathfrak{A}_{m}\left(2 r^{2}-8 x r+4 x^{2} ; x\right) \\
= & \mathfrak{A}_{m}\left(4 r^{2} ; x\right)-8 x \mathfrak{A}_{m}(r ; x)+4 x^{2} \mathfrak{A}_{m}(1 ; x) \\
= & 4\left[\mathfrak{A}_{m}\left(r^{2} ; x\right)-x^{2}\right]-8 x\left[\mathfrak{A}_{m}(r ; x)-x\right] \\
& +4 x^{2}\left[\mathfrak{A}_{m}(1 ; x)-1\right] .
\end{aligned}
$$

Using (3.11), we obtain

$$
\begin{aligned}
\mathfrak{A}_{m}(f ; x)-f(x)< & \varepsilon \mathfrak{A}_{m}(1 ; x)+\frac{2 \mathcal{N}}{\delta^{2}}\left\{4\left[\mathfrak{A}_{m}\left(r^{2} ; x\right)-x^{2}\right]\right. \\
& \left.-8 x\left[\mathfrak{A}_{m}(r ; x)-x\right]+4 x^{2}\left[\mathfrak{A}_{m}(1 ; x)-1\right]\right\} \\
& +f(x)\left[\mathfrak{A}_{m}(1 ; x)-1\right] . \\
= & \varepsilon\left[\mathfrak{A}_{m}(1 ; x)-1\right]+\varepsilon+\frac{2 \mathcal{N}}{\delta^{2}}\left\{4\left[\mathfrak{A}_{m}\left(r^{2} ; x\right)-x^{2}\right]\right. \\
& \left.-8 x\left[\mathfrak{A}_{m}(r ; x)-x\right]+4 x^{2}\left[\mathfrak{A}_{m}(1 ; x)-1\right]\right\} \\
& +f(x)\left[\mathfrak{A}_{m}(1 ; x)-1\right] .
\end{aligned}
$$

Since $\varepsilon>0$ is arbitrary, we can write

$$
\begin{aligned}
\left|\mathfrak{A}_{m}(f ; x)-f(x)\right| \leqq & \varepsilon+\left(\varepsilon+\frac{8 \mathcal{N}}{\delta^{2}}+\mathcal{N}\right)\left|\mathfrak{A}_{m}(1 ; x)-1\right| \\
& +\frac{16 \mathcal{N}}{\delta^{2}}\left|\mathfrak{A}_{m}(r ; x)-x\right|+\frac{8 \mathcal{N}}{\delta^{2}}\left|\mathfrak{A}_{m}\left(r^{2} ; x\right)-x^{2}\right| \\
\leqq & \mathcal{E}\left(\left|\mathfrak{A}_{m}(1 ; x)-1\right|+\left|\mathfrak{A}_{m}(r ; x)-x\right|\right. \\
& \left.+\left|\mathfrak{A}_{m}\left(r^{2} ; x\right)-x^{2}\right|\right),
\end{aligned}
$$


where

$$
\mathcal{E}=\max \left(\varepsilon+\frac{8 \mathcal{N}}{\delta^{2}}+\mathcal{N}, \frac{16 \mathcal{N}}{\delta^{2}}, \frac{8 \mathcal{N}}{\delta^{2}}\right)
$$

Now, for a given $\mu>0$, there exists $\varepsilon>0(\varepsilon<\mu)$, we get

$$
\mathfrak{G}_{m}(x ; \mu)=\left\{m: m \leqq P_{n} \quad \text { and } \quad p_{m}\left|\mathfrak{A}_{m}(f ; x)-f(x)\right| \geqq \mu\right\} .
$$

Furthermore, for $k=0,1,2$, we have

$$
\mathfrak{G}_{k, m}(x ; \mu)=\left\{m: m \leqq P_{n} \quad \text { and } \quad p_{m}\left|\mathfrak{A}_{m}(f ; x)-f_{k}(x)\right| \geqq \frac{\mu-\varepsilon}{3 \mathcal{E}}\right\},
$$

so that,

$$
\mathfrak{G}_{m}(x ; \mu) \leqq \sum_{k=0}^{2} \mathfrak{G}_{k, m}(x ; \mu)
$$

Clearly, we obtain

$$
\frac{\left\|\mathfrak{G}_{m}(x ; \mu)\right\|_{\mathcal{C}[0,1]}}{P_{n}} \leqq \sum_{k=0}^{2} \frac{\left\|\mathfrak{G}_{k, m}(x ; \mu)\right\|_{\mathcal{C}[0,1]}}{P_{n}} .
$$

Now, using the above assumption about the implications in (3.2) to (3.4) and by Definition 4, the right-hand side of (3.13) is seen to tend to zero as $n \rightarrow \infty$. Consequently, we get

$$
\lim _{n \rightarrow \infty} \frac{\left\|\mathfrak{G}_{m}(x ; \mu)\right\|_{\mathcal{C}[0,1]}}{P_{n}}=0(\delta, \mu>0) .
$$

Therefore, implication (3.1) holds true. This completes the proof of Theorem 3.

Next, by using Definition 5, we present the following theorem.

Theorem 4. Let $\mathfrak{A}_{m}: \mathcal{C}[0,1] \rightarrow \mathcal{C}[0,1]$ be a martingale difference sequence of positive linear operators and let $f \in \mathcal{C}[0,1]$. Then

$$
\operatorname{stat}_{\text {DWMD }} \lim _{m \rightarrow \infty}\left\|\mathfrak{A}_{m}(f ; x)-f(x)\right\|_{\infty}=0
$$

if and only if

$$
\begin{aligned}
& \text { stat }_{\text {DWMD }} \lim _{m \rightarrow \infty}\left\|\mathfrak{A}_{m}(1 ; x)-1\right\|_{\infty}=0, \\
& \text { stat }_{\text {DWMD }} \lim _{m \rightarrow \infty}\left\|\mathfrak{A}_{m}(2 x ; x)-2 x\right\|_{\infty}=0
\end{aligned}
$$

and

$$
\operatorname{stat}_{\text {DMD }} \lim _{m \rightarrow \infty}\left\|\mathfrak{A}_{m}\left(3 x^{2} ; x\right)-3 x^{2}\right\|_{\infty}=0 .
$$

Proof. The proof of Theorem 4 is similar to the proof of Theorem 3. We, therefore, choose to skip the details involved. 
We present below an illustrative example for the martingale difference sequence of positive linear operators that does not satisfy the conditions of the weighted statistical convergence versions of Korovkin-type approximation Theorem 3 and also the results of Srivastava et al. [22], and Paikray et al. [11], but it satisfies the conditions of statistical weighted summability versions of our Korovkin-type approximation Theorem 4. Thus, our Theorem 4 is stronger than the results asserted by Theorem 3 and also, the results of Srivastava et al. [22] and Paikray et al. [11].

We now recall the operator

$$
\vartheta(1+\vartheta D) \quad\left(D=\frac{d}{d \vartheta}\right)
$$

which was used by Al-Salam [1] and, more recently, by Viskov and Srivastava [25] (see [14] and the monograph by Srivastava and Manocha [24] for various general families of operators and polynomials of this kind). Here, in our Example 3 below, we use this operator in conjunction with the Bernstein polynomial.

Example 3. We consider Bernstein polynomial $\mathfrak{B}_{m}(f ; \vartheta)$ on $C[0,1]$ given by

$$
\mathfrak{B}_{m}(f ; \vartheta)=\sum_{m=0}^{n} f\left(\frac{m}{n}\right)\left(\begin{array}{c}
n \\
m
\end{array}\right) \vartheta^{m}(1-\vartheta)^{n-m} \quad(\vartheta \in[0,1]) .
$$

Next, we present the martingale difference sequences of positive linear operators on $C[0,1]$ defined as follows:

$$
\mathfrak{A}_{m}(f ; \vartheta)=\left[1+\left(Y_{n}, \mathcal{F}_{n}\right)\right] \vartheta(1+\vartheta D) \mathfrak{B}_{m}(f ; \vartheta) \quad(\forall f \in C[0,1]),
$$

where $\left(Y_{n}, \mathcal{F}_{n}\right)$ is already mentioned in Example 2.

Now, we calculate the values of the functions $1,2 \vartheta$ and $3 \vartheta^{2}$ by using our proposed operators (3.19),

$$
\begin{aligned}
\mathfrak{A}_{m}(1 ; \vartheta) & =\left[1+\left(Y_{m}, \mathcal{F}_{m}\right)\right] \vartheta(1+\vartheta D) 1=\left[1+\left(Y_{m}, \mathcal{F}_{m}\right)\right] \vartheta \\
\mathfrak{A}_{m}(2 \vartheta ; \vartheta) & =\left[1+\left(X_{m}, \mathcal{F}_{m}\right)\right] \vartheta(1+\vartheta D) 2 \vartheta=\left[1+\left(Y_{m}, \mathcal{F}_{m}\right)\right] \vartheta(1+2 \vartheta),
\end{aligned}
$$

and

$$
\begin{aligned}
\mathfrak{A}_{m}\left(3 \vartheta^{2} ; \vartheta\right) & =\left[1+\left(Y_{m}, \mathcal{F}_{m}\right)\right] \vartheta(1+\vartheta D) 3\left\{\vartheta^{2}+\frac{\vartheta(1-\vartheta)}{m}\right\} \\
& =\left[1+\left(Y_{m}, \mathcal{F}_{m}\right)\right]\left\{\vartheta^{2}\left(6-\frac{9 \vartheta}{m}\right)\right\},
\end{aligned}
$$

so that we have

$$
\begin{aligned}
& \operatorname{stat}_{\text {DWMD }} \lim _{m \rightarrow \infty}\left\|\mathfrak{A}_{m}(1 ; \vartheta)-1\right\|_{\infty}=0, \\
& \operatorname{stat}_{\text {DWMD }} \lim _{m \rightarrow \infty}\left\|\mathfrak{A}_{m}(2 \vartheta ; \vartheta)-2 \vartheta\right\|_{\infty}=0
\end{aligned}
$$

and

$$
\operatorname{stat}_{\text {DWMD }} \lim _{m \rightarrow \infty}\left\|\mathfrak{A}_{m}\left(3 \vartheta^{2} ; \vartheta\right)-3 \vartheta^{2}\right\|_{\infty}=0
$$


Consequently, the sequence $\mathfrak{A}_{m}(f ; \vartheta)$ satisfies the conditions (3.15) to (3.17). Therefore, by Theorem 4, we have

$$
\operatorname{stat}_{\text {DWMD }} \lim _{m \rightarrow \infty}\left\|\mathfrak{A}_{m}(f ; \vartheta)-f\right\|_{\infty}=0 .
$$

Here, the given martingale difference sequence $\left(Y_{m}, \mathcal{F}_{m}\right)$ of functions in Example 2 is statistically deferred weighted summable but not deferred weighted statistically convergent. Thus, martingale difference operators defined by (3.19) satisfy the Theorem 4; however, it is not satisfying Theorem 3 .

Moreover, if one considers the positive linear operators of the types Baskakov and Szász-Mirakyan [6], and Beta Szász-Mirakjan [16] in place of Bernstein polynomial $\mathfrak{B}_{m}(f ; \vartheta)$ in Example 3, then with the same algebraic test functions it will also satisfy the conclusion of Korovkin-type approximation theorem via our purposed mean for martingale difference sequences of random variables. Consequently, these operators are also valid for Theorem 4; however, it will not satisfy Theorem 3.

\section{Concluding Remarks and ObSERVATIONS}

In this concluding section of our investigation, we present several further remarks and observations concerning to various results which we have proved here.

Remark 1. Let $\left(Y_{n}, \mathcal{F}_{n} ; n \in \mathbb{N}\right)$ be a martingale difference sequence given in Example 2. Then, since

$$
\text { stat }_{\text {DWMD }} \lim _{m \rightarrow \infty} Y_{m}=0 \text { on }[0,1],
$$

we have

$$
\text { stat }_{\text {DWMD }} \lim _{m \rightarrow \infty}\left\|\mathfrak{A}_{m}\left(f_{k} ; x\right)-f_{k}(x)\right\|_{\infty}=0 \quad(k=0,1,2) .
$$

Thus, by Theorem 4, we can write

$$
\operatorname{stat}_{\text {DWMD }} \lim _{m \rightarrow \infty}\left\|\mathfrak{A}_{m}(f ; x)-f(x)\right\|_{\infty}=0,
$$

where

$$
f_{0}(x)=1, \quad f_{1}(x)=2 x \quad \text { and } \quad f_{2}(x)=3 x^{2} .
$$

Here, the martingale difference sequence $\left(Y_{n}, \mathcal{F}_{n} ; n \in \mathbb{N}\right)$ is neither statistically convergent nor converges uniformly in the ordinary sense; thus, the classical and statistical versions of Korovkin-type theorems do not work here for the operators defined by (3.19). Hence, this application indicates that our Theorem 4 is a non-trivial generalization of the classical as well as statistical versions of Korovkin-type theorems (see [5] and [10]).

Remark 2. Let $\left(Y_{n}, \mathcal{F}_{n} ; n \in \mathbb{N}\right)$ be a martingale difference sequence given already in Example 2. Then, since

$$
\text { stat }
$$


so (4.1) holds true. Now, by applying (4.1) and Theorem 4, condition (4.2) also holds true. However, since the martingale difference sequence $\left(Y_{n}, \mathcal{F}_{n} ; n \in \mathbb{N}\right)$ is not deferred weighted statistically convergent but it is statistically deferred weighted summable. Thus, Theorem 4 is certainly a non-trivial extension of Theorem 3. Therefore, Theorem 4 is stronger than Theorem 3.

Conflicts of Interest: The authors declare that they have no conflicts of interest.

\section{REFERENCES}

[1] W. A. Al-Salam, "Operational representations for the Laguerre and other polynomials." Duke Math. J., vol. 31, pp. 127-142, 1964, doi: 10.1215/S0012-7094-64-03113-8.

[2] N. L. Braha, V. Loku, and H. M. Srivastava, " $\Lambda^{2}$-Weighted statistical convergence and Korovkin and Voronovskaya type theorems." Appl. Math. Comput., vol. 266, pp. 675-686, 2015.

[3] N. L. Braha, H. M. Srivastava, and S. A. Mohiuddine, "A Korovkin-type approximation theorem for periodic functions via the statistical summability of the generalized de la Vallée Poussin mean." Appl. Math. Comput., vol. 228, pp. 162-169, 2014.

[4] A. A. Das, S. K. Paikray, T. Pradhan, and H. Dutta, "Statistical $(C, 1)(E, \mu)$-summablity and associated fuzzy approximation theorems with statistical fuzzy rates." Soft Comput., vol. 24, pp. 10 883-10 892, 2020, doi: 10.1007/s00500-019-04591-2.

[5] H. Fast, "Sur la convergence statistique." Colloq. Math., vol. 2, pp. 241-244, 1951.

[6] V. Gupta, A. M. Acu, and H. M. Srivastava, "Difference of some positive linear approximation operators for higher-order derivatives." Symmetry, vol. 12, pp. 1-19, 2020, doi: 10.3390/sym 12060915.

[7] B. B. Jena and S. K. Paikray, "Product of statistical probability convergence and its applications to Korovkin-type theorem." Miskolc Math. Notes, vol. 20, pp. 969-984, 2019, doi: 10.18514/MMN.2019.3014.

[8] B. B. Jena, S. K. Paikray, and H. Dutta, "On various new concepts of statistical convergence for sequences of random variables via deferred Cesàro mean.” J. Math. Anal. Appl., vol. 487, pp. 1-18, 2020, doi: 10.1016/j.jmaa.2020.123950.

[9] B. B. Jena, S. K. Paikray, S. A. Mohiuddine, and V. N. Mishra, "Relatively equi-statistical convergence via deferred Nörlund mean based on difference operator of fractional-order and related approximation theorems." AIMS Math., vol. 5, pp. 650-672, 2020, doi: 10.3934/math.2020044.

[10] P. P. Korovkin, "Convergence of linear positive operators in the spaces of continuous functions (in Russian).” Doklady Akad. Nauk. SSSR (New Ser.), vol. 90, pp. 961-964, 1953.

[11] S. K. Paikray and H. Dutta, Applied Mathematical Analysis: Theory, Methods, and Applications: On statistical deferred weighted $\mathcal{B}$-convergence. Springer Nature Switzerland AG: Springer, 2019. doi: 10.1007/978-3-319-99918-0_20.

[12] P. Parida, S. K. Paikray, and B. B. Jena, "Generalized deferred Cesàro equi-statistical convergence and analogous approximation theorems," Proyecciones J. Math., vol. 39, pp. 307-331, 2020, doi: 10.22199.

[13] T. Pradhan, S. K. Paikray, B. B. Jena, and H. Dutta, "Statistical deferred weighted $\mathcal{B}$-summability and its applications to associated approximation theorems." J. Inequal. Appl., vol. 2018, pp. 1-21, 2018, doi: 10.1186/s13660-018-1650-x.

[14] H. M. Srivastava, "A note on certain operational representations for the Laguerre polynomials." $J$. Math. Anal. Appl., vol. 138, pp. 209-213, 1989, doi: 10.1016/0022-247X(89)90331-4.

[15] H. M. Srivastava and M. Et, "Lacunary statistical convergence and strongly lacunary summable functions of order $\alpha . ”$ Filomat, vol. 31, pp. 1573-1582, 2017. 
[16] H. M. Srivastava, G. İçöz, and B. Çekim, "Approximation properties of an extended family of the Szász-Mirakjan Beta-type operators." Axioms, vol. 8, pp. 1-13, 2019, doi: 10.3390/axioms8040111.

[17] H. M. Srivastava, B. B. Jena, and S. K. Paikray, "Deferred Cesàro statistical convergence of martingale sequence and Korovkin-type approximation theorems." Miskolc Math. Notes, to be published.

[18] H. M. Srivastava, B. B. Jena, and S. K. Paikray, "Deferred Cesàro statistical probability convergence and its applications to approximation theorems." J. Nonlinear Convex Anal., vol. 20, pp. 1777-1792, 2019.

[19] H. M. Srivastava, B. B. Jena, and S. K. Paikray, "A certain class of statistical probability convergence and its applications to approximation theorems." Appl. Anal. Discrete Math., vol. 14, pp. 579-598, 2020, doi: doi.org/10.2298/AADM190220039S.

[20] H. M. Srivastava, B. B. Jena, and S. K. Paikray, "Statistical deferred Nörlund summability and Korovkin-type approximation theorem." Mathematics, vol. 8, pp. 1-11, 2020, doi: 10.3390/math8040636.

[21] H. M. Srivastava, B. B. Jena, and S. K. Paikray, "Statistical probability convergence via the deferred Nörlund mean and its applications to approximation theorems." Rev. R. Acad. Cienc. Exactas Fís. Nat. Ser. A Mat. (RACSAM), vol. 114, pp. 1-14, 2020, doi: 10.1007/s13398-020-008757.

[22] H. M. Srivastava, B. B. Jena, S. K. Paikray, and U. K. Misra, "Generalized equi-statistical convergence of the deferred Nörlund summability and its applications to associated approximation theorems." Rev. R. Acad. Cienc. Exactas Fís. Nat. Ser. A Mat. (RACSAM), vol. 112, pp. 14871501, 2018, doi: 10.1007/s13398-017-0442-3.

[23] H. M. Srivastava, B. B. Jena, S. K. Paikray, and U. K. Misra, "Statistically and relatively modular deferred-weighted summability and Korovkin-type approximation theorems." Symmetry, vol. 11, pp. 1-20, 2019, doi: 10.3390/sym11040448.

[24] H. M. Srivastava and H. L. Manocha, A Treatise on Generating Functions. New York, Chichester, Brisbane and Toronto: John Wiley and Sons, 1984.

[25] O. V. Viskov and H. M. Srivastava, "New approaches to certain identities involving differential operators." J. Math. Anal. Appl., vol. 186, pp. 1-10, 1994, doi: 10.1006/jmaa.1994.1281.

[26] A. Zraiqat, S. K. Paikray, and H. Dutta, "A certain class of deferred weighted statistical $B$ summability involving $(p, q)$-integers and analogous approximation theorems." Filomat, vol. 33, pp. 1425-1444, 2019.

Authors' addresses

Bidu Bhusan Jena

Department of Mathematics, Veer Surendra Sai University of Technology, Burla 768018, Odisha, India

E-mail address: bidumath.05@gmail.com

\section{Susanta Kumar Paikray}

Department of Mathematics, Veer Surendra Sai University of Technology, Burla 768018, Odisha, India

E-mail address: skpaikray math@vssut.ac.in 